Editorial

\title{
Translational Research in Urology Training
}

\section{Investigación Traslacional en Formación en Urología}

\author{
Leonardo O. Reis ${ }^{1}$ \\ ${ }^{1}$ Livre Docente Urologia, Universidade de Campinas, São Paulo, Brazil \\ Urol Colomb 2019;28:273-274.
}

In a continuum aimed at improving population health, translational medicine must bridge the gap between basic research and clinical needs. There are two main stages of translational research, the transfer of new knowledge from the laboratory to human trials and from these clinical studies to health practice and decision making. ${ }^{1}$

For doctors, the most important question is how to treat the patient, with the study of the disease being a secondary target, important only to improve the treatment of future patients. The opposite is often true for scientists, who will be taught by clinical exposition about the pressures and time constraints of clinical medicine.

In the surgical context of Urology, due to time constraints for bench work, translational research is limited to the mastery of basic scientists or academic surgeons with protected research hours, and even clinical trials are difficult for a surgeon to administer and case series, with their known limitations and biases, are the likely scientific output of most surgeons.

In addition, far from adequately measuring intermediate or long-term research results, such as the adoption of new therapeutic and diagnostic practices, changes in public policy or improvements in population health, researchers and research institutions generally evaluate the impact of translational research by observing easily determinable measures such as the publication of articles in high impact factor specialized journals, success in the acquisition of research grants and obtaining patents registries.

As a sequel, from a flurry of research work, a very small fraction translates into real benefit to patients, which is often limited, when it occurs. Among several aspects related to this phenomenon, results demonstrated in cell culture and animal models may have super-optimistic interpretations that are not valid in humans. ${ }^{2}$

Although short-term results may be observed in translation, such as improvements in the research process, others may require some years, while some depend on the long term, for example, changes in medical practice, development of guidelines, and drug approval. Indeed, demonstrable impacts on individuals and communities caused by translational research such as improved health, disease rates, and improvements in health care costs may not manifest themselves anytime soon.

On average more than 20 years are required for the translational research process to impact human life. One of the most prestigious prizes of all time in the world, the Nobel Prize “... for those who ... bestowed the greatest benefit to humanity ..." captures the real sense of this and as early as November 27, 1895, Alfred Nobel in his third and last will at the Swedish-Norwegian Paris Club recognized the importance of translational research that he called physiology or medicine, “... a part for the person who made the most important discovery in the field of physiology or medicine. .. $" 3$.

It is important to note that works that motivate the Nobel Prize in physiology or medicine are not always published in a prestigious journal with a high impact factor, a practice that corresponds to the apex of the current science paradigm.

Doctoral programs in biomedical sciences have traditionally directed scientists to narrow and specialized careers, without providing exposure and training on how to translate their research into meaningful clinical findings and post doctors and junior teachers, with or without clinical training, who have already completed their doctorate degrees, could benefit greatly from training in clinical and translational research. $^{2,4}$

Just as physicians need the assistance of experienced "pure" scientists for experimental laboratory activities, they rely on the help of clinicians to explore the critical real-world context, as the clinical concerns that limit translation are often not known to scientists. Only by understanding clinical disease and standard treatment paradigms can a scientist identify unmet clinical needs and design clinically relevant translation experiments.

While understanding the molecular, cellular, and clinical pathophysiology of the disease is essential for successful translational research, with increasing patient care information, there is a temptation to truncate basic science in the

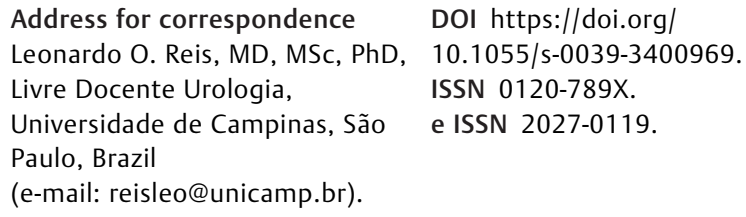

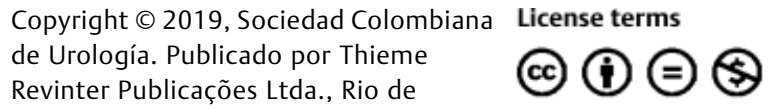

Janeiro, Brazil. Todos los derechos reservados. 
medical curriculum. Medical school courses do not explore the latest in-depth experimental methods and technologies and generally do not provide detailed instructions for the development and conduct of clinical trials. ${ }^{4}$

Some universities, including Brazilians, offer the MD/PhD Medical Scientist program in which the doctoral component is usually packaged against a traditional doctoral program and some $\mathrm{MD} / \mathrm{PhD}$ training experiences may not be as rich as a traditional program that is about discovery and experience as well as specific knowledge, one of the reasons that doctoral training is usually not timed. In addition, most PhD scientists gain 3-5 years of postdoctoral research experience before starting their own independent research.

Medical scientists must gain in-depth knowledge of at least their subspecialty to establish and conduct an optimal translational research program, and throughout the research continuum, from planning, implementation, evaluation, dissemination to translation for the benefit of the population, ethical issues are fundamental.

Ironically, Brazil has one of the greatest and most emblematic examples of medical scientist in history. Between 1909 and 1921, the physician Carlos Ribeiro Justiniano das Chagas, a young research team leader, was able to describe the etiology, vector, reservoir, morphological characteristics, acute, chronic stages and clinical aspects of a new disease, besides raising the possibility of autoimmunity in its pathogenesis, characterizing the most complete translational discovery of all time. Chagas received the Schaudinn Prize in 1912 in Europe and competed with more than 80 scientists for the 1921 Nobel Prize which was curiously not offered that year. ${ }^{5}$

Medical scientists, though play a central role in translational medicine, account for less than $1 \%$ of doctors in the US, driven by many factors including little salaries, which in academic medical centers are generally lower compared with private practice, and they may be in the laboratory only part-time, even though competing with full-time scientists for funding. Because doctors often generate more clinical revenue than research income for an institution, there is constant pressure to increase clinical time/performance. ${ }^{6}$

To reverse the growing deficit of translational research leaders, we must redefine metrics that value meritocracy based on solid long-term results, for beyond publication in prestigious journals with high impact factors, acquisition of research funding, and registering patents, which are not directly responsible for the success of translation; linked to mechanisms and policies for the promotion of team science that identify, train and retain PhD scientists with a background in clinical and translational sciences, living the challenges of medicine.

\section{References}

1 Choi PJ, Tubbs RS, Oskouian RJ. The Current Trend of the Translational Research Paradigm. Cureus 2018;10(03):e2340

2 Comeau DL, Alvarez JA, Bhatti P, et al. Building Diverse Careers in Clinical and Translational Research: Evaluation of a Certificate Program in Translational Research. J Clin Transl Sci 2018;2(01): 38-47

3 Nobel Prize. (2019). Accessed: November 7, 2019: https://www. nobelprize.org/alfred-nobel/alfred-nobels-will/

4 Translational science spectrum. (2019). Accessed: November 7, 2019:https://ncats.nih.gov/translation

5 Bestetti RB, Cardinalli-Neto A. Dissecting slander and crying for justice: Carlos Chagas and the Nobel Prize of 1921. Int J Cardiol 2013;168(03):2328-2334

6 Pickering CR, Bast RC Jr, Keyomarsi K. How will we recruit, train, and retain physicians and scientists to conduct translational cancer research? Cancer 2015;121(06):806-816 